\title{
Memory destabilization is critical for the success of the reactivation-extinction procedure
}

\author{
Marcelo E. Piñeyro, ${ }^{1,3}$ Roque I. Ferrer Monti, ${ }^{1,3}$ Joaquín M. Alfei, ${ }^{1}$ Adrián M. Bueno, ${ }^{1}$ \\ and Gonzalo P. Urcelay ${ }^{2,4}$ \\ ${ }^{1}$ Laboratorio de Psicología Experimental, Facultad de Psicología, Universidad Nacional de Córdoba, 5000 Córdoba, Argentina; \\ ${ }^{2}$ Department of Psychology \& Behavioural and Clinical Neuroscience Institute, University of Cambridge, Cambridge CB2 3EB, \\ United Kingdom
}

\begin{abstract}
It has been suggested that, unlike pure extinction which typically results in the return of the fear response under a variety of circumstances, memory reactivation followed by extinction can attenuate the reemergence of conditioned fear. The reactivation-extinction procedure has attracted the attention of basic and clinical researchers due to its potential clinical value for the treatment of psychiatric conditions, such as anxiety and drug abuse disorders. However, mixed results have been achieved so far in replicating and understanding this paradigm. It has been proposed that memory destabilization could be critical in this sense. Using contextual fear conditioning in rats and midazolam as an amnesic agent, we first determined what reactivation conditions are necessary to destabilize the mnemonic trace. After establishing the conditions for memory destabilization, a series of experiments was conducted to determine if destabilization is critical for the success of the reactivation-extinction procedure. Data confirmed the importance of memory destabilization prior to extinction inside the reconsolidation window to attenuate spontaneous recovery and retard reacquisition of conditioned fear. The present report offers a candidate explanation of the discrepancy in results obtained with the reactivation-extinction procedure by different laboratories.
\end{abstract}

Exposure therapies are widely used for the treatment of anxiety disorders, and are based on extinction learning which can be explored in controlled experimental conditions inside the laboratory (Urcelay 2012). In both clinical and experimental settings, conditioned responses decrease through repeated noncontingent presentations of the conditioned stimulus (i.e., extinction) and there is considerable evidence suggesting long-term improvements in clinical populations after exposure therapy (Choy et al. 2007). However, responding reappears under a wide variety of circumstances, all of which involve a contextual change between extinction and subsequent testing (Mineka et al. 1999; Rodríguez et al. 1999; Mystkowski et al. 2002; Bouton 2004). This observation has led to the suggestion that extinction involves new learning and memory formation, rather than erasure of the original association (Bouton 2002). In recent years, however, it has been recognized that previously consolidated memories can be destabilized, if reactivated under appropriate conditions (Finnie and Nader 2012), and thus become vulnerable to the effects of amnesic agents. This opens a possibility for long-term intervention of traumatic memories which underlie anxiety disorders (Diergaarde et al. 2008).

Reactivation can be defined as any process that triggers a stored memory into an active state (Lewis 1979) and is a prerequisite of memory retrieval (Gisquet-Verrier and Riccio 2012). Memory destabilization, on the other side, is difficult to identify because its occurrence may not be related to any particular behavioral manifestation, although some molecular processes have been thought to mediate memory destabilization, such as synaptic protein degradation (Lee et al. 2008). In order to persist, a de-

\footnotetext{
${ }^{3}$ These authors contributed equally to this work.

${ }^{4}$ Corresponding author

E-mail gu203@cam.ac.uk

Article is online at http://www.learnmem.org/cgi/doi/10.1101/Im.032714.113.
}

stabilized memory must become stable again (i.e., restabilize or reconsolidate).

Though the conditions necessary to destabilize a memory through its reactivation are not always met, when they are reactivation induces a transient destabilization of the memory (also termed labile or unstable phase), making it vulnerable to pharmacological interference and behavioral manipulations. It follows that not every reactivation treatment induces memory destabilization and it is important to differentiate both constructs. While unstable, the memory is vulnerable to interference, and this limited time period is termed the "reconsolidation window." Accordingly, to determine if a specific reactivation procedure destabilizes a particular memory, interference procedures must alter memory expression when applied inside the reconsolidation window. The same procedure applied outside this time period should have no effect on memory expression (Nader and Hardt 2009).

The fact that a long-term memory can return to a malleable or unstable form through reactivation has many theoretical, practical, and clinical implications, hence efforts to understand this post-reactivation plasticity process (broadly termed "reconsolidation") have grown exponentially in the last decade (Besnard et al. 2012). For example, in a recent report, Monfils et al. (2009) revealed a promising approach for the treatment of fear-related memories based on the manipulation of the reconsolidation process followed immediately by extinction (Monfils et al. 2009). Using auditory fear conditioning in rats, they found evidence of attenuation of conditioned fear expression by reactivating the memory through presentation of a single conditioned stimulus

\footnotetext{
C 2013 Piñeyro et al. This article is distributed exclusively by Cold Spring Harbor Laboratory Press for the first 12 months after the full-issue publication date (see http://learnmem.cshlp.org/site/misc/terms.xhtml). After 12 months, it is available under a Creative Commons License (AttributionNonCommercial 3.0 Unported), as described at http://creativecommons. org/licenses/by-nc/3.0/.
} 
(CS-reactivation) followed 10 or $60 \mathrm{~min}$ later by extinction training of the reactivated CS. The effects of this manipulation were evaluated using standard tests for relapse: spontaneous recovery, context renewal, and reinstatement (Bouton 2004). Results showed no reemergence of the fear response to the CS under reactivation-extinction conditions. However, pure extinction or extinction applied outside the reconsolidation window $(6 \mathrm{~h})$ showed the typical recovery effects in those same tests. In addition, this manipulation rendered the CS-US association harder to retrain (i.e., retardation of reacquisition), compared to control groups. Schiller et al. (2010) replicated this finding in human fear conditioning and observed attenuated recovery of the fear response even after a year. Similarly, Xue et al. (2012) found analogous results using rats in a drug conditioned place preference paradigm, as well as when exposing heroin addicts to drug-related stimuli.

Findings such as those of Monfils et al. (2009) and Schiller et al. (2010) could have implications for psychotherapy. Memory destabilization through CS-reactivation followed by extinction within the reconsolidation window represents a unique opportunity to attenuate relapse in a purely behavioral, noninvasive way. This is perhaps the most promising clinical application of basic research findings on the process of reconsolidation. Indeed, attempts to replicate and understand this procedure readily appeared, in both humans and animals alike, with mixed success (for discussion, see Hutton-Bedbrook and McNally 2013). Some studies report positive results (Clem and Huganir 2010; Schiller et al. 2010; Flavell et al. 2011; Rao-Ruiz et al. 2011; Agren et al. 2012a, b; Ma et al. 2012; Oyarzún et al. 2012; Xue et al. 2012; Zhang et al. 2012) while others failed to replicate the effect (Pérez-Cuesta and Maldonado 2009; Chan et al. 2010; Costanzi et al. 2011; Soeter and Kindt 2011; Golkar et al. 2012; Ishii et al. 2012; Kindt and Soeter 2013). In a recent review (Auber et al. 2013) it was suggested that subtle methodological differences could explain these discrepancies. One possibility, the authors argue, is that negative results are due to a failure to destabilize the memory through CS-reactivation. If the reactivation procedure does not induce memory destabilization, then responding will recover under any of the traditional challenges to extinction learning. It follows that, according to Auber et al. (2013), there is a crucial role of memory destabilization in the success of the reactivation-extinction procedure.

We hypothesized that the relationship between the amount of CS-reactivation and memory destabilization need not be monotonic. As it is the case in standard conditioning (Fig. 1; see Rescorla 1988), one would expect that too little exposure to the CS during reactivation would not be sufficient to destabilize the memory. Some optimal amount of reactivation would be needed to successfully induce destabilization, whereas too much reactivation would induce extinction (Lee et al. 2006). Thus if memory destabilization is to have an inverted $U$ relationship with the amount of reactivation, then too little reactivation will not induce memory destabilization, and too much will induce extinction.

We decided to address these concerns experimentally, using contextual fear conditioning in rats, as it offers a simple way to manipulate the destabilization process through the duration of the reactivation session. In this preparation it has been consistently reported that following short reactivations (i.e., $1 \mathrm{~min}$ ) amnesic agents fail to disrupt reconsolidation, while longer reactivations (3-5 min) induce memory destabilization and therefore reconsolidation, allowing interference by pharmacological means (Suzuki et al. 2004; Lee et al. 2008; Bustos et al. 2009).

In Experiment 1, we searched for the optimal reactivation interval to destabilize a contextual fear memory. Different groups received context fear training and $3 \mathrm{~d}$ later were exposed to the context for different intervals, followed by a systemic injection of midazolam (MDZ), a fast-acting GABA-A receptor agonist that has been reported to disrupt contextual fear reconsolidation (Bustos et al. 2009, 2010). In Experiment 2, we tested a longer amount of reactivation that would lead to standard extinction, resulting in significant freezing reduction when tested $24 \mathrm{~h}$ later and spontaneous recovery after $1 \mathrm{wk}$. Therefore, Experiments 1 and 2 established the optimal parameters to reactivate, destabilize, or extinguish the fear memory. Experiment 3 a tested if mere reactivation $(1 \mathrm{~min})$ and later extinction would reduce spontaneous recovery after $1 \mathrm{wk}$, while Experiment $3 \mathrm{~b}$ addressed a similar question but destabilizing the memory through reactivation (4 min) before extinction. The results revealed that memory destabilization must be achieved through reactivation for the extinction procedure to attenuate spontaneous recovery of the conditioned response. Experiment 4 demonstrated that extinction training outside the reconsolidation window ( $6 \mathrm{~h}$ later), opened by a 4-min destabilizing reactivation session, behaves like regular extinction, showing spontaneous recovery a week later.

Finally, Experiment 5 was designed to replicate the main findings of Experiment 3, but rather than assessing spontaneous recovery, we investigated whether the procedure would result in retarded reemergence of behavioral control with retraining (i.e., test of reacquisition). If so, it would reveal that memory destabilization before applying extinction is critical to retard reemergence of the fear response in addition to attenuated spontaneous 
recovery, thus adding generality to our conclusions. In addition, in this experiment we included several control groups to avoid cross-experiment comparisons. Thus, one group received normal extinction, a second group received mere reactivation followed by extinction, the critical group received destabilizing memory reactivation and extinction, and a fourth control group received no extinction at all. The results replicated previous findings showing that only memory destabilization followed later by extinction was able to retard reacquisition. Hence, we conclude that memory destabilization is a prerequisite for the reactivation-extinction procedure to attenuate spontaneous recovery from extinction and retard reacquisition.

\section{Results}

\section{Experiment 1}

This first experiment was designed to establish the optimal parameters that lead to memory destabilization through a reactivation session in our laboratory. It has been reported by different groups (and with different amnesic agents) that duration of the reactivation trial is crucial in the dynamics of the destabilization and reconsolidation of contextual fear memories: that is, short reactivations (i.e., $1 \mathrm{~min}$ ) are not enough to destabilize the memory trace, while longer reactivations (3-5 $\mathrm{min}$ ) are necessary for amnesic agents to block the reconsolidation of a putative destabilized memory (Suzuki et al. 2004; Lee et al. 2008; Bustos et al. 2009). Therefore, $72 \mathrm{~h}$ after context fear conditioning, rats were exposed for different amounts of time to the training context $(1,4$, or 5 $\mathrm{min}$ ) and immediately injected with $3 \mathrm{mg} / \mathrm{kg} \mathrm{MDZ}$, a dose previously reported to block memory reconsolidation in this preparation (Bustos et al. 2009), or saline vehicle (SAL). The possible amnesic effects of MDZ per se were controlled by adding a fourth condition with subjects receiving only MDZ or SAL, but no memory reactivation (control group). The effects of these manipulations were evaluated $24 \mathrm{~h}$ later through a 5 -min test session, during which rats were exposed to the context in the absence of shock.

Figure 1A depicts the experimental protocol. Figure 1, B and $\mathrm{C}$, shows freezing during reactivation and test, respectively. There were no significant differences between groups during reactivation $(P>0.05$ in all cases). A factorial ANOVA (drug $\times$ reactivation duration) on the test data revealed a significant effect of drug $\left.F_{(1,40)}=21.1, P<0.01\right)$, a significant effect of reactivation duration $\left(F_{(3,40)}=15.0, P<0.01\right)$, and a significant drug $\times$ reactivation interaction $\left(F_{(3,40)}=12.9, P<0.01\right)$. Post hoc analysis revealed that MDZ and SAL groups differed only when reactivation lasted 4 or $5 \mathrm{~min}$, while no differences were found for the control groups (no reactivation) or when reactivation was of $1 \mathrm{~min}$.

These results suggest various conclusions: First, the benzodiazepine agent MDZ lacks any amnesic effect when administered alone or after a very brief ( $1 \mathrm{~min}$ ) reactivation, as previously reported (Bustos et al. 2009). A very different result emerges when reactivation is extended up to $4-5 \mathrm{~min}$, since freezing behavior is substantially reduced at test. This pattern is in agreement with other reports using different drugs (Suzuki et al. 2004; Lee et al. 2008) and MDZ (Bustos et al. 2009). According to Lee et al. (2008), synaptic protein degradation (a putative molecular mechanism of memory destabilization) does not occur in contextual fear conditioning without memory reactivation or when reactivation is too brief (i.e., $1 \mathrm{~min}$ ). When reactivation is extended in duration, then destabilization occurs, and the mnemonic trace needs to restabilize or reconsolidate, a process which can be experimentally manipulated. In line with this reasoning, our results show that mere reactivation does not necessarily mean that the memory will destabilize and therefore reconsolidate. Reactivation must fulfill, at the very least, a minimum duration requirement, and under our experimental conditions, this requirement is set at $4 \mathrm{~min}$ of exposure. Since we did not find any differences in the amnesic effects of MDZ between 4- and 5-min reactivations, we decided to use 1and 4-min reactivations in subsequent experiments. This provides us with the possibility to simply reactivate $(1 \mathrm{~min})$ or destabilize (4 min) the contextual fear memory, without unnecessarily extending the exposure duration to the conditioned context, which could theoretically lead to extinction learning.

\section{Experiment 2}

Since the procedure proposed by Monfils et al. (2009) is based on the assumption that the new extinction learning interferes or updates a destabilized memory trace, we needed to establish an extinction protocol able to generate such a new memory (i.e., Context-noUS). This new extinction memory should fulfill at least two conditions: First, it should be present at least $24 \mathrm{~h}$ after training, in order to guarantee that it is a long-term memory; the second condition is that, like any extinction memory, the conditioned response should recover spontaneously over the passage of time (Bouton 2002).

Figure 2A depicts the experimental protocol. Two groups of rats were conditioned as in Experiment 1, and $72 \mathrm{~h}$ later one group (extinction) was exposed to the training context for $15 \mathrm{~min}$, without shock. The other group (control) did not receive any context exposure. Both groups received a 5-min test $24 \mathrm{~h}$ after extinction training, as in Experiment 1, and both groups were again evaluated $7 \mathrm{~d}$ later in identical conditions to assess spontaneous recovery (retest).

Figure $2 \mathrm{~B}$ shows the extinction training data (extinction group only), while Figure $2 \mathrm{C}$ shows results for test and retest (both groups). A repeated measures ANOVA was conducted for the extinction group alone on total freezing during extinction training. This was achieved by dividing the 15 -min extinction session into three 5-min bins ( $0-5,5-10$, and $10-15 \mathrm{~min})$. A repeated measures ANOVA revealed a significant effect of time $\left(F_{(2,14)}=\right.$ $6.0475, P<0.05)$. Post hoc analysis revealed that freezing behavior decreased significantly from $0-5$ to the 5-10 intervals, and remained stable through the last 10-15 $\mathrm{min}$. In order to compare both groups during test and retest, we conducted a repeated measures ANOVA (group $\times$ evaluation phase), that yielded a significant effect of group $\left(F_{(1,14)}=7.76, P<0.05\right)$, no effect of evaluation phase $\left(F_{(1,14)}<1\right)$, but a significant group $\times$ evaluation phase interaction $\left(F_{(1,14)}=7.30, P<0.05\right)$. To analyze the source of this interaction, post hoc analyses were conducted. These revealed that both groups differed during test (i.e., extinction), but not during retest $7 \mathrm{~d}$ later (i.e., spontaneous recovery). At the same time, the control group did not differ between test and retest, but the extinction group did: There was a significant recovery in freezing from test to retest $(P<0.05)$.

These results show that 15 min of extinction training is sufficient to reduce the conditioned response during extinction training and $24 \mathrm{~h}$ later, thus indicating the existence of a longterm extinction memory. However, this reduction recovers $7 \mathrm{~d}$ later, which is the typical spontaneous recovery effect following extinction. In essence, this experiment confirms the widely accepted notion that extinction learning recovers with the passage of time (Bouton 2004).

\section{Experiment 3a}

Considering the results of Experiments 1 and 2, we have established parameters leading to reactivation (1 min), destabilization (4 min), or extinction (15 min) of a contextual fear memory. Experiments $3 \mathrm{a}$ and $3 \mathrm{~b}$ were designed to test the hypothesis that 


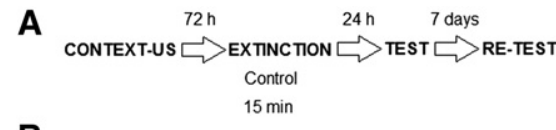

B

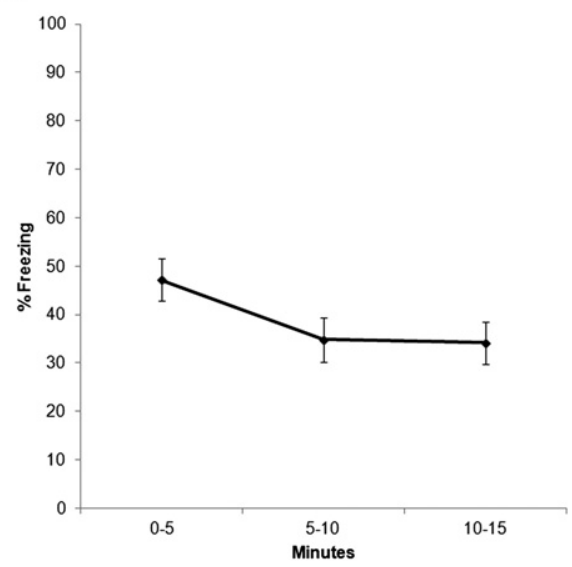

C

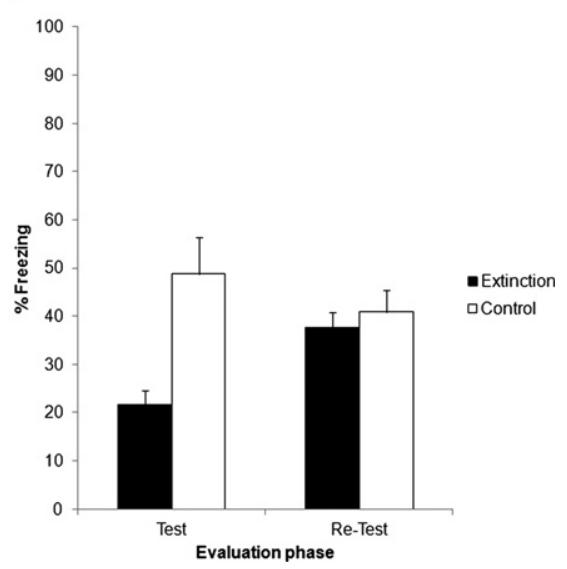

Figure 2. Experiment 2. (A) Experimental protocol. Seventy-two hours after contextual fear conditioning, rats were exposed to training context for $15 \mathrm{~min}$ without shock (extinction group) or remained untreated (control group). Both groups were evaluated $24 \mathrm{~h}$ later in a 5 -min test session and again $7 \mathrm{~d}$ later in a retest session. $(B)$ Data represent the mean \pm SEM of percentage time spent freezing during extinction training for the extinction group. $(C)$ Data showing the mean $\pm S E M$ of percentage time spent freezing during test and retest, for both groups.

memory destabilization is critical for the success of the procedure reported by Monfils et al. (2009). In order to make the experiments comparable, we designed our studies so that the total duration of context exposure was always $15 \mathrm{~min}$ in the critical groups: in Experiment 3a, a 1-min reactivation was followed by 14 min of extinction, whereas in Experiment $3 \mathrm{~b} 4 \mathrm{~min}$ of reactivation were followed by $11 \mathrm{~min}$ of extinction. If memory destabilization is critical for the effectiveness of subsequent extinction, we predicted to observe attenuated recovery $7 \mathrm{~d}$ later only in the group that received 4 min of reactivation prior to extinction.

In Experiment 3a, two groups of rats were fear conditioned and $72 \mathrm{~h}$ later both received a 1-min reactivation session. Immediately after reactivation, animals of both groups were returned to home cages. One group was reexposed to the training context for 14 min (group reactivation 1 min plus extinction 14 min or R1/E14) 30 min after reactivation. The second group served as a control and remained in the home cage, not receiving any further context exposure (group R1/E0). Both groups were evaluated in a 5 -min test session $24 \mathrm{~h}$ later and $7 \mathrm{~d}$ later in identical conditions, as in Experiment 2. Figure 3A depicts the experimental protocol.

Figure $3 \mathrm{~B}$ shows the results of reactivation, test, and retest phases for both groups. As expected, there were no differences between groups during reactivation $(P>0.05)$. A repeated measures ANOVA for test and retest data (group $\times$ evaluation phase as factors) revealed a significant effect of group $\left(F_{(1,13)}=11.62, P<\right.$ $0.01)$, no effect of evaluation phase $\left(F_{(1,13)}=3.80, P>0.07\right)$, and a significant interaction between both factors $\left(F_{(1,13)}=\right.$ $14.96, P<0.01)$. Post hoc analyses revealed that groups differed during test but not during retest. In other words, the difference was lost 7 d later. Finally, group R1/E14 expressed significantly more freezing from test to retest (i.e., spontaneous recovery).

These results are in agreement with our hypothesis. Although the contextual fear memory was reactivated and later extinguished, spontaneous recovery was observed 1 wk later. This pattern should not be observed if reactivation is prolonged to $4 \mathrm{~min}$, which is precisely what Experiment $3 \mathrm{~b}$ was designed to examine.

\section{Experiment $3 b$}

According to Experiment 1, to destabilize a contextual fear memory under our experimental conditions, 4 min of reactivation are required. It follows, then, that adding 11 min of extinction training half-an-hour later (thus completing a total of $15 \mathrm{~min}$ of context exposure) should reduce the conditioned response $24 \mathrm{~h}$ later, and sustain such a reduction 1 wk later (in other words, attenuated spontaneous recovery should be observed), because, according to Monfils et al. (2009), memory destabilization is a prerequisite for extinction training applied inside the reconsolidation window to alter the original CS-US association.

Two groups of rats were trained and $72 \mathrm{~h}$ later reactivated, like in Experiment $3 a$, except that this time reactivation lasted 4 min and the extinction training applied $30 \mathrm{~min}$ later lasted $11 \mathrm{~min}$. Figure $4 \mathrm{~A}$ shows the experimental protocol. Accordingly, groups were named R4/ E11 and R4/E0. Test and retest were identical to those in Experiment 3a.

Figure 4B shows the results of reactivation, test, and retest phases for both groups. No difference was found between groups during reactivation $(P>0.05)$. A repeated measures ANOVA on test and retest data (group $\times$ evaluation phase as factors) revealed a significant effect of group $\left(F_{(1,14)}=32.84, P<0.01\right)$, and no significant effect

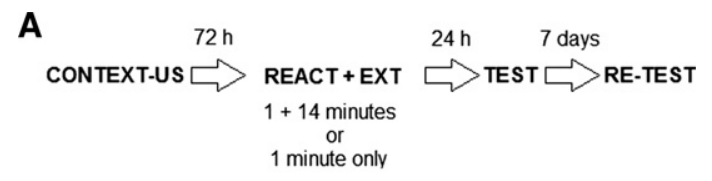

B

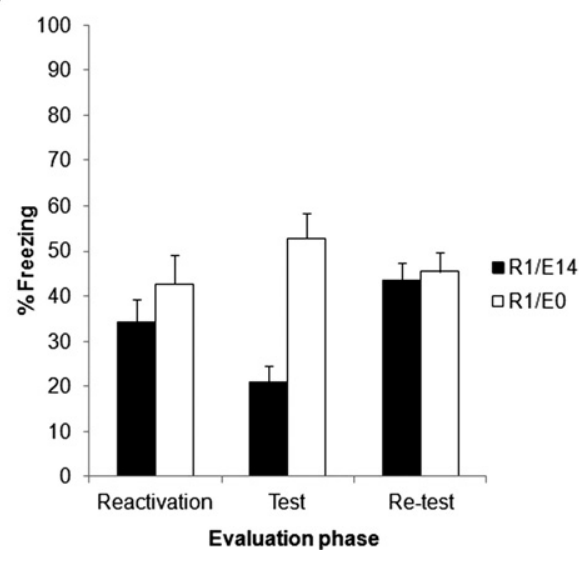

Figure 3. Experiment 3a. (A) Experimental protocol. Seventy-two hours after contextual fear conditioning, rats were exposed to the training context for $1 \mathrm{~min}$. Thirty minutes later, half of the rats were returned to the training context for a 14-min extinction session (group R1/E14). The remaining half did not receive any further treatment beyond the 1 -min reactivation and served as controls (group R1/E0). Both groups were evaluated $24 \mathrm{~h}$ later in a 5-min test and again $7 \mathrm{~d}$ later in a retest.

(B) Data depict the mean \pm SEM of percentage time spent freezing during reactivation, test, and retest. 


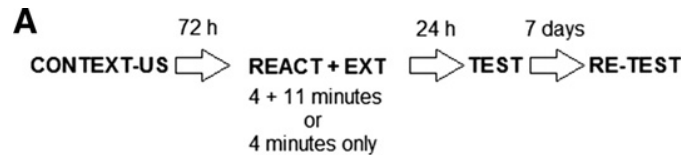

B

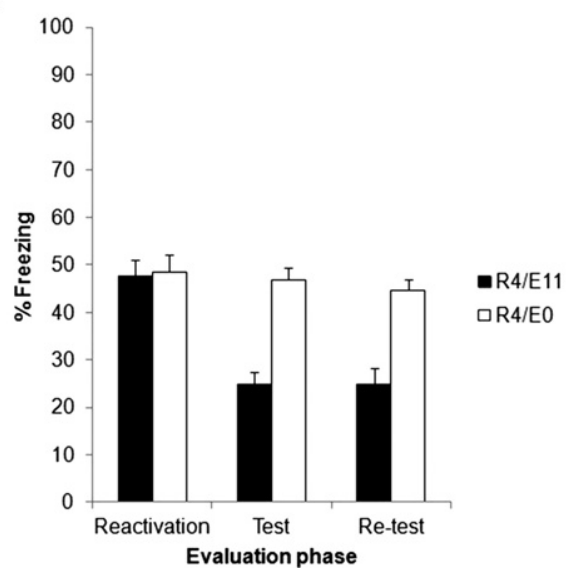

Figure 4. Experiment 3b. (A) Experimental protocol. Seventy-two hours after contextual fear conditioning, rats were exposed to the training context for $4 \mathrm{~min}$. Thirty minutes later, one-half of the rats were returned to the training context for an 11-min extinction session (group R4/E11). The other half did not receive any further treatment beyond the 4 min of reactivation and served as controls (group R4/E0). Both groups were evaluated $24 \mathrm{~h}$ later in a 5-min test and again $7 \mathrm{~d}$ later in a retest. (B) Data show the mean \pm SEM of percentage time spent freezing during reactivation, test, and retest.

of evaluation phase $\left(F_{(1,14)}=0.46, P>0.05\right)$ or interaction $\left(F_{(1,14)}=0.46, P>0.05\right)$. Post hoc analyses revealed that groups differed between them during both test and retest. Hence, no spontaneous recovery was observed $7 \mathrm{~d}$ later.

These results are in agreement with those obtained in Experiment 1 in that 4 min is sufficient to induce memory destabilization through reactivation. But critically, the only difference between Experiments 3a and 3b was that reactivation was increased from 1 to $4 \mathrm{~min}$ before completing, $30 \mathrm{~min}$ later, a total of 15 min of context exposure in the absence of shock. Spontaneous recovery was observed in the former case and absent in the latter.

There is ample evidence demonstrating that memory destabilization and later reconsolidation are time-limited processes (Nader and Hardt 2009). A 4-min reactivation followed later by 11 min of extinction should not attenuate recovery if extinction learning occurs when memory destabilization is outside of the reconsolidation window. Using similar parameters to those used in the present experiments, and MDZ as amnesic agent, Bustos et al. (2006) observed that the reconsolidation window of contextual fear memories closes $2 \mathrm{~h}$ after reactivation. Accordingly, in Experiment 4, we hypothesized that a 4-min reactivation followed $6 \mathrm{~h}$ later by $11 \mathrm{~min}$ of extinction learning should not attenuate spontaneous recovery, similar to normal extinction without reactivation (Experiment 2) or when memory destabilization is not achieved through reactivation (Experiment 3a).

\section{Experiment 4}

One group was used for this experiment. Rats received fear conditioning and $72 \mathrm{~h}$ later reactivated during $4 \mathrm{~min}$ as in Experiment $3 \mathrm{~b}$, except that the $11 \mathrm{~min}$ of extinction training took place $6 \mathrm{~h}$ later, rather than $30 \mathrm{~min}$ later, based on the assumption that memory destabilization would have ceased by that time. Figure
$5 \mathrm{~A}$ depicts the experimental protocol. Figure 5B shows the results of reactivation, test, and retest sessions. A repeated measures ANOVA on test and retest data (evaluation phase as factor) revealed a significant effect of revaluation phase $\left(F_{(1,7)}=59.78\right.$, $P<0.01)$. Post hoc analyses demonstrated that there was a significant freezing increase from test to retest.

Although this experiment did not assess a full temporal gradient between reactivation and extinction on spontaneous recovery, this result is consistent with previous reports in suggesting that the reconsolidation process takes no more than $2 \mathrm{~h}$ in contextual fear memories (Bustos et al. 2006). Accordingly, if extinction learning takes place once this limited time period is over, spontaneous recovery should not be attenuated, which is what was observed in Experiment 4.

\section{Experiment 5}

There are numerous treatments that result in recovery from extinction (Urcelay 2012), but we decided to test reacquisition, that is the reemergence of responding that occurs when the CS (i.e., context) is paired again with the US once extinction has already taken place. That was the aim of this final experiment. Moreover, besides adding generality to the findings of Experiments $3 \mathrm{a}$ and $3 \mathrm{~b}$, we wanted to document the effect in a single experiment including all relevant controls. Figure 6A depicts the experimental protocol. Rats were trained as in previous experiments and $72 \mathrm{~h}$ later assigned randomly to one of four groups: extinction (R0/E15), control (R0/E0), R1/E14, or R4/ E11. Treatments for each group were identical to those in previous experiments (no exposure, 15 full minutes, or divided in $1 / 14$ or $4 / 11)$. Twenty-four hours after context exposure in the groups receiving extinction, all groups were again exposed to the training context for $3 \mathrm{~min}$ (preshock period) after which they received a single $0.5-\mathrm{mA}$ shock. The number and intensity of shock was reduced to $50 \%$ relative to the original training to avoid ceiling effects of fear responding during test that could mask differences between

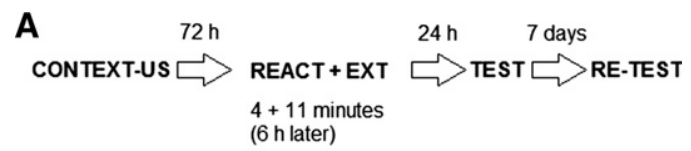

B

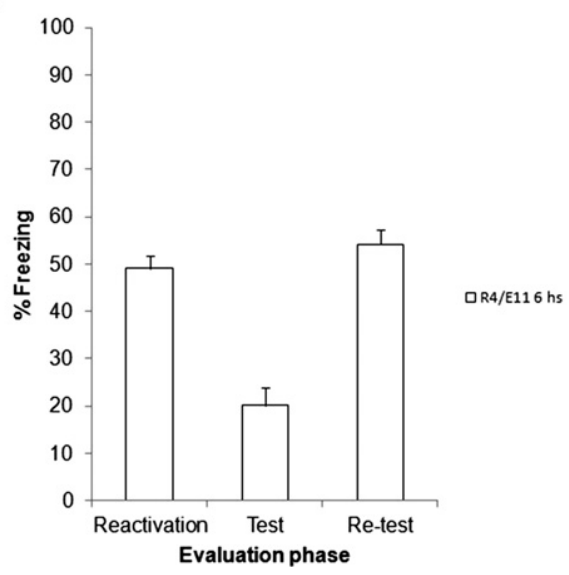

Figure 5. Experiment 4. (A) Experimental protocol. Seventy-two hours after contextual fear conditioning, rats were exposed to the training context for $4 \mathrm{~min}$. Six hours later, rats were returned to the training context for an 11-min extinction session (R4/E11 6 h). Rats were evaluated $24 \mathrm{~h}$ later in a 5-min test and again $7 \mathrm{~d}$ later in a retest. (B) Data are the mean \pm SEM of percentage time spent freezing during reactivation, test, and retest. 


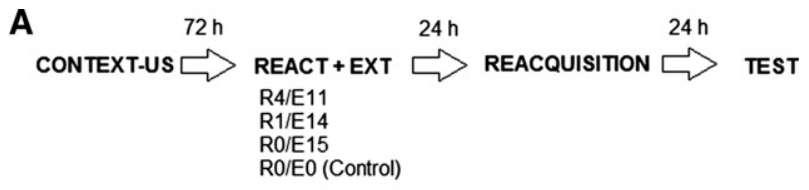

B

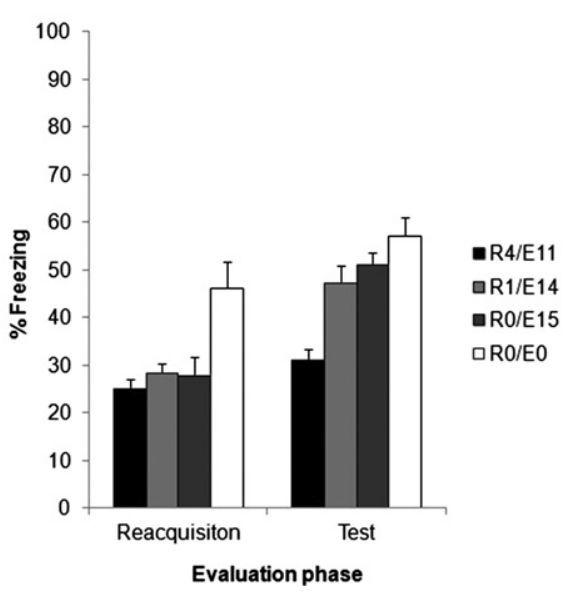

Figure 6. Experiment 5. (A) Experimental protocol. Rats were trained as in former experiments and $72 \mathrm{~h}$ later assigned randomly to one of four groups: extinction (R0/E15), control (R0/E0), R1/E14, or R4/E11. Treatments for each group were identical to those in previous experiments. Twenty-four hours later, all groups were again exposed to the training context for $3 \mathrm{~min}$ (preshock period) after which they received a single 0.5-mA shock. Twenty-four hours later all groups were subjected to a 5 -min test session. (B) Data are the mean \pm SEM of percentage time spent freezing during the preshock period of reacquisition and test.

treatments, since rapid and strong reacquisition (i.e., savings) occurs when the reinforcer is presented again in the original training context (Bouton 2002). Twenty-four hours later all groups were tested as in previous experiments. In order to test our prediction that retarded reemergence should only be observed in Group R4/E11, groups were compared in two instances: during the preshock period of the reacquisition session (all groups had already been extinguished under different regimes, except of course those in the control group) and during the test $24 \mathrm{~h}$ after reacquisition. Figure 6A depicts the experimental protocol.

Figure $6 \mathrm{~B}$ shows the results of reacquisition and test phases. A one-way ANOVA (group as factor) on the preshock period of the reacquisition session revealed a significant effect of group $\left(F_{(3,25)}=6.6195, P<0.01\right)$. Post hoc analyses revealed that the control group was the only one that differed from all others, with no differences among experimental groups. This is expected because the control group was the only one not subjected to any extinction procedure, hence high levels of freezing persist during this stage. A one-way ANOVA (group as factor) on the test signaled a significant effect of group $\left(F_{(3,25)}=11.099, P<0.01\right)$. Post hoc analyses demonstrated that only group R4/E11 differed from the other groups, thus showing attenuated reacquisition of behavioral control.

\section{Discussion}

The major finding of the present study is that memory destabilization is a prerequisite for the beneficial effects of the reactivationextinction procedure described by Monfils et al. (2009). This is, to our knowledge, the first report to reveal one reason why many studies around the world have failed to obtain such a benefit, with some studies even reporting the opposite effect (Chan et al. 2010; for review, see Auber et al. 2013). Results from several experiments support this conclusion. Experiment 1 showed that the vulnerability of the contextual fear memory to the amnesic effect of MDZ is observed after either 4 or 5 min of reactivation, but not when 1 min of reactivation is used. A possible explanation for this result is that $1 \mathrm{~min}$ is not sufficient to destabilize the trace through initiation of synaptic protein degradation, a process that can take place after at least 3 or $5 \mathrm{~min}$, as demonstrated by Lee et al. (2008) and also indirectly shown by others in contextual fear with rodents (Suzuki et al. 2004; Bustos et al. 2009). Experiment 2 showed that $15 \mathrm{~min}$ of extinction training substantially reduces freezing behavior during extinction and $24 \mathrm{~h}$ later, but that this effect recovers after $7 \mathrm{~d}$. A very similar pattern emerges when 1-min reactivation is administered half-an-hour before 14 min of extinction, as observed in Experiment 3a. Both procedures led to a decrease in freezing behavior that recovered 7 d later. However, Experiment 3b revealed that when 4-min reactivation (which induces memory destabilization, as observed in Experiment 1) preceded 11 min of extinction, spontaneous recovery was attenuated.

As was observed in Experiment 4, when extinction was administered $6 \mathrm{~h}$ after reactivation (that is, outside of the reconsolidation window), recovery was again observed. Finally, Experiment 5 replicated and extended these findings by showing that destabilization followed by extinction attenuates reacquisition, at least under the present experimental conditions. This adds generality to the findings of Experiments 3a and 3b, while comparing all relevant groups in one experiment. Our results are in agreement with those of Monfils et al. (2009) and the interpretations of Auber et al. (2013), because the effect is assumed to occur under a crucial premise: Prior memory destabilization is a critical process for extinction to be durable and resistant to recovery, which we fully confirmed in this study. Moreover, these results demonstrate one variable which seems to be critical for the observation of the effect, the amount of exposure during reactivation, since $1 \mathrm{~min}$ of reactivation did not produce such a robust extinction, thus offering a candidate explanation for the numerous failed attempts to replicate the original report. We do not argue that the duration of the reactivation is the only and critical variable. Indeed, Clem and Huganir (2010) observed that the effectiveness of the reactivation-extinction procedure is highly dependent on the conditioning-reactivation interval, as the effect was observed when this interval was $1 \mathrm{~d}$ but not $7 \mathrm{~d}$ (in the current experiments it was $3 \mathrm{~d}$ ). Rather, we suggest that since trace destabilization cannot be directly detected, it must be inferred from an experimental manipulation in order to assure its occurrence. The fact that in contextual fear conditioning reactivation duration seems to be critical to induce trace destabilization, as seen by Suzuki et al. (2004), Lee et al. (2008) and Bustos et al. (2009), suggests that different preparations and parametric variations may probably require different reactivation conditions to induce trace destabilization. The critical issue is to determine precisely which conditions are necessary in each case, and our results suggest that, everything else being equal, the duration of exposure can have a critical effect.

Soeter and Kindt (2011) using a differential fear conditioning paradigm in humans, and employing propanolol as an amnesic agent to reveal the occurrence of the destabilization and reconsolidation processes, did not provide support for the reactivationextinction procedure. These results seem to be at odds with the current findings. However, as the authors suggested, the number of extinction trials utilized in their study was perhaps insufficient to induce extinction. In agreement with this view, in Experiment 2 they observed spontaneous recovery for the control CS as soon as $24 \mathrm{~h}$ after completing extinction training, an effect that was attenuated in the reactivated CS condition. Therefore, it is 
reasonable to agree with the author's interpretation: Although the memory was destabilized (which is deduced by the amnesic effects of propanolol), the amount of extinction training was insufficient to induce memory updating. Hence, in Experiment 2 of the present research our aim was to obtain an extinction protocol strong enough to reduce the conditioned response at least $24 \mathrm{~h}$ after training, since this would guarantee the existence of a long-term extinction memory, thus avoiding the complication reported by Soeter and Kindt (2011).

Although procedures that maximize extinction learning are a promising avenue of research, it is hitherto unclear why some laboratories failed to obtain durable effects with reactivation-extinction procedures, while others succeeded. A parsimonious interpretation assumes that the relationship between reactivation and memory destabilization forms an inverted U-shaped function. Too little reactivation would not induce memory destabilization, whereas too much reactivation would trigger an extinction process. In both cases, extinction applied later would be less capable of interfering with the original memory, and less durable effects should be expected. As stated earlier, memory destabilization cannot be directly observed. It must be shown to be taking place by pharmacological manipulation under particular reactivation conditions. A logical inference, then, is that equating mere reactivation to memory destabilization could lead to erroneous conclusions.

A number of major questions remain unanswered and should be addressed in order to comprehend and manipulate the reactivation-extinction procedure in a clinical setting. The first one is, of course, what conditions need/must be met in order to destabilize a memory? Or, simply put, when does reconsolidation occur? Large efforts are being made to answer that question (for review, see Finnie and Nader 2012), but recent experimental data in both rats (Lee 2008; Díaz-Mataix et al. 2013) and humans (Sevenster et al. 2012, 2013) suggest that prediction error or a mismatch between what is expected and what is experienced should take place in order for memories to undergo reconsolidation. That hypothesis was already advanced by Maldonado's group a few years earlier (Pedreira et al. 2004) and by Eisenberg et al. (2003). It is tempting to conclude that the reactivation conditions necessary to induce trace destabilization in this study produced a mismatch between training and reactivation, since shock was delivered at $3 \mathrm{~min}$ (and again $30 \mathrm{sec}$ later) during conditioning, while no shock was delivered in a similar amount of time (4 min) during reactivation. Consistent with this interpretation, unpublished data from our laboratory suggest that prediction error may be key to destabilization.

In summary, we believe that the potential of procedures that exploit both reconsolidation and extinction (Monfils et al. 2009; Schiller et al. 2010) are enormously rich because they avoid pharmacological intervention. Despite numerous failures (for review, see Auber et al. 2013), the phenomenon has been revealed in different species and experimental preparations, and clinical applications are feasible given the successes in humans. The present report clarifies these discrepancies by showing a critical variable (amount of exposure, assumed to reveal destabilization only after sufficient reactivation) that determines whether the benefit on extinction learning occurs or not.

\section{Materials and Methods}

\section{Subjects}

Subjects were experimentally naive, adult male Wistar rats (60 to 65-d old, weighing 270-320 g at the beginning of the experiments). Animals were bred in our colony in the Laboratorio de Psicología Experimental, Facultad de Psicología, Universidad Nacional de Córdoba, Argentina. All animals were housed in stan- dard laboratory Plexiglas cages $(60 \mathrm{~cm}$ long $\times 40 \mathrm{~cm}$ wide $\times 20 \mathrm{~cm}$ high) in groups of $3-4$ per cage. Food and water were available ad libitum. Animals were maintained on a 12-h light/dark cycle (lights on at $8 \mathrm{a} . \mathrm{m}$.), at room temperature of $21^{\circ} \mathrm{C}-23^{\circ} \mathrm{C}$. The standards of the NIH Guide for the Care and Use of Laboratory Animals were respected. The number of animals and their suffering was kept to the minimum possible to achieve the goals of this research.

\section{Drugs}

Midazolam (MDZ, Gobbi Novag SA) was diluted in sterile isotonic saline (SAL, $0.9 \% \mathrm{w} / \mathrm{v}$ ) to a concentration of $3 \mathrm{mg} / \mathrm{mL}$, and administered intraperitoneally (i.p.). The total volume of drug or equivalent amount of SAL was $1.0 \mathrm{~mL} / \mathrm{kg}$ in all cases. This dose of MDZ was selected on the basis of previous reports demonstrating its ability to block contextual fear memory reconsolidation in Wistar rats (Bustos et al. 2009).

\section{Apparatus}

Contextual fear conditioning was conducted in a $24 \mathrm{~cm}$ long $\times 22$ $\mathrm{cm}$ wide $\times 22 \mathrm{~cm}$ high Plexiglas chamber with opaque gray walls and a removable transparent ceiling, the floor consisting of 20 parallel stainless-steel grid bars, each measuring $3 \mathrm{~mm}$ in diameter, spaced $1 \mathrm{~cm}$ apart, and connected to a device to provide adjustable foot shocks (Automatic Reflex Conditioner 7501, Ugo Basile). The chamber was cleaned with water and dried with paper towels before and after all subjects. Recording of behavior (for offline analysis) was made with a DCR-SR21 Sony Handycam digital video camera placed $50 \mathrm{~cm}$ above the conditioning chamber. Background noise was supplied with ventilation fans. All procedures were made in a sound-isolated experimental room. Experiments were always performed during the light phase of the cycle.

\section{Behavioral procedures}

In all experiments, rats were first identified, weighed, and handled for 5 min on two separate days to habituate them to experimental manipulation. In those experiments involving i.p. injections, rats were also injected with $1 \mathrm{~mL} / \mathrm{kg}$ SAL after handling was complete to habituate them to this procedure.

\section{Contextual fear conditioning}

One day after habituation procedures ended, rats were taken out individually from their home cage, transported into the experimental room, and exposed to the conditioning chamber for 3 min (preshock period), after which two foot shocks $(1.0 \mathrm{~mA}$, 3 -sec duration, with an inter-shock interval of $30 \mathrm{sec}$ ) serving as USs were delivered. Immediately after the second shock ended, rats were removed from the chamber, transported back to the colony room, and placed back in their home cages.

\section{Reactivation session}

Reactivations were always carried out $72 \mathrm{~h}$ after conditioning. Rats were reexposed to the conditioning chamber, without foot shock, for different periods of time $(1,4$, or $5 \mathrm{~min})$, depending on the experiment.

\section{Drug administration}

In Experiment $1, \mathrm{MDZ} 3 \mathrm{mg} / \mathrm{kg}$ or an equivalent amount of SAL was injected i.p. immediately after reactivation sessions.

\section{Extinction}

Extinction consisted of exposing rats to the conditioning chamber during 11, 14, or $15 \mathrm{~min}$, depending on the experiment. However, in all groups receiving reactivation and extinction, the total amount of context exposure was always 15 min (whether it was 15 min straight or divided in $1 / 14$ or $4 / 11$ ). 


\section{Reacquisition}

In Experiment 5 reacquisition consisted of a 3-min preshock period followed by only one 3-sec shock of $0.5 \mathrm{~mA}$ (half the number [2] and intensity [1.0] of US compared to initial conditioning), after which rats were immediately removed from the chamber and taken back to their home cages in the colony room.

Test

Tests consisted of a 5-min exposure session to the conditioning chamber, without shock.

Retest

Retests were identical to test, but $7 \mathrm{~d}$ later.

\section{Behavioral scoring}

All experiments were video-taped for later offline analyses. Freezing behavior, defined as the total absence of body and head movements except for that associated with breathing (Blanchard and Blanchard 1969), was scored minute-by-minute with a stopwatch by an observer blind to the experimental condition of each animal, and expressed as percent of time (in seconds). Inter-observer reliability was established with a different set of data (Pearson's $r=0.95$ ).

\section{Experiment 1}

Rats were first subjected to a contextual fear conditioning. Seventy-two hours later, they were randomly assigned to one of four reactivation conditions, depending on reactivation trial duration, 1, 4, or $5 \mathrm{~min}$, and a control group (no reactivation at all or $0 \mathrm{~min}$ ). Immediately after reactivation, half of the rats in each condition received a $3 \mathrm{mg} / \mathrm{kg} \mathrm{MDZ}$ injection (i.p.) and the other half received an equivalent amount of SAL. Groups were labeled according to drug (MDZ or SAL) and reactivation trial duration $(0,1,4$, or $5 \mathrm{~min})$. Twenty-four hours after reactivation, all groups were subjected to a 5-min test. For all groups, $n=6$.

\section{Experiment 2}

Seventy-two hours after conditioning, half of the rats were submitted to a 15-min extinction session and the other half served as controls (no context exposure). Number of subjects: extinction $(n=8)$ and control $(n=8)$. Both groups were submitted to a test and retest $24 \mathrm{~h}$ and $7 \mathrm{~d}$ after the extinction session, respectively.

\section{Experiment 3a}

Seventy-two hours after conditioning, all rats were submitted to a 1 -min reactivation session. Immediately after reactivation, rats were transported back to their home cages. Half of the rats were returned to the experimental room $30 \mathrm{~min}$ after reactivation and were reexposed to the conditioning chamber for $14 \mathrm{~min}$ and then returned back to their home cages, while the other half were not. Groups sizes were as follows: reactivation of 1 min and extinction of $14 \mathrm{~min}$ or $\mathrm{R} 1 / \mathrm{E} 14(n=7)$ and reactivation of $1 \mathrm{~min}$ and extinction of $0 \mathrm{~min}$ or R1/E0 $(n=8)$. Both groups were submitted to a test and retest, identical to Experiment 2.

\section{Experiment $3 b$}

Identical to Experiment 3a, except that the reactivation session lasted $4 \mathrm{~min}$ and the extinction session lasted $11 \mathrm{~min}$. Group sizes were as follows: R4/E11 $(n=8)$ and R4/E0 $(n=8)$. Both groups were submitted to a test and retest, identical to Experiments 2 and $3 a$.

\section{Experiment 4}

Identical to Experiment 3b, except that the time between reactivation and extinction sessions was $6 \mathrm{~h}$, not $30 \mathrm{~min}$ : R4/E11 $(n=8)$.

\section{Experiment 5}

Rats were first subjected to contextual fear conditioning and $72 \mathrm{~h}$ later randomly assigned to one of four groups: extinction $(n=8)$, control $(n=7), \mathrm{R} 1 / \mathrm{E} 14(n=7)$, or R4/E11 $(n=7)$. Groups extinction and control were identical to Experiment 2, while groups R1/ E14 and R4/E11 to Experiments 3a and 3b, respectively. Twentyfour hours after completing treatment, all groups were subjected to a reacquisition session, consisting in a 3 -min preshock period followed by a single 0.5 -mA shock lasting $3 \mathrm{sec}$. Twenty-four hours after completing reacquisition, all groups were subjected to a 5 -min test session.

\section{Statistical analyses}

Results were expressed as mean \pm SEM of the percentage time the animal spent freezing. Significant ANOVAs were followed by the Newman-Keuls Test for post hoc analysis. In all cases, $P<0.05$ was the statistical threshold.

\section{Acknowledgments}

This work was supported by a grant from Secretaría de Ciencia y Técnica (SeCyT) from the Universidad Nacional de Córdoba, Argentina, to A.M.B. M.E.P. was supported by a pre-graduate SeCyT studentship. R.I.F.M. was supported by a doctoral SeCyT studentship. We thank Zara Y. Goozée and Dr. Victor A. Molina for valuable comments on earlier versions of the manuscript and the reviewers of this study for their insightful and helpful critiques and observations.

\section{References}

Agren T, Engman J, Frick A, Björkstrand J, Larsson E, Furmark T, Fredrikson M. 2012a. Disruption of reconsolidation erases a fear memory trace in the human amygdala. Science 337: 1550-1552.

Agren T, Furmark T, Eriksson E, Fredrikson M. 2012b. Human fear reconsolidation and allelic differences in serotonergic and dopaminergic genes. Transl Psychiatry 2: 1-6.

Auber A, Tedesco V, Jones C, Monfils M, Chiamulera C. 2013. Post-retrieval extinction as reconsolidation interference: Methodological issues or boundary conditions? Psychopharmacology 226: 631-647.

Besnard A, Caboche J, Laroche S. 2012. Reconsolidation of memory: A decade of debate. Prog Neurobiol 99: 61-80.

Blanchard R, Blanchard D. 1969. Passive and active reactions to fear-eliciting stimuli. J Comp Physiol Psychol 68: 129-135.

Bouton M. 2002. Context, ambiguity, and unlearning: Sources of relapse after behavioral extinction. Biol Psychiatry 52: 976-986.

Bouton M. 2004. Context and behavioral processes in extinction. Learn Mem 11: 485-494.

Bustos S, Maldonado H, Molina V. 2006. Midazolam disrupts fear memory reconsolidation. Neuroscience 139: 831-842.

Bustos S, Maldonado H, Molina V. 2009. Disruptive effect of midazolam on fear memory reconsolidation: Decisive influence or reactivation time span and memory age. Neuropsychopharmacology 34: 446-457.

Bustos S, Giachero M, Maldonado H, Molina V. 2010. Previous stress attenuates the susceptibility to midazolam's disruptive effect on fear memory reconsolidation: Influence of pre-reactivation D-cycloserine administration. Neuropsychopharmacology 35: 1097-1108.

Chan W, Leung H, Westbrook R, McNally G. 2010. Effects of recent exposure to a conditioned stimulus on extinction of pavlovian fear conditioning. Learn Mem 17: 512-521.

Choy Y, Fyer A, Lipsitz J. 2007. Treatment of specific phobia in adults. Clin Psychol Rev 27: 266-286.

Clem R, Huganir R. 2010. Calcium-permeable AMPA receptor dynamics mediate fear memory erasure. Science 330: 1108-1112.

Costanzi M, Cannas S, Saraulli D, Rossi-Arnaud C, Cestari V. 2011. Extinction after retrieval: Effects on the associative and nonassociative components of remote contextual fear memory. Learn Mem 18: 508-518.

Díaz-Mataix L, Ruiz Martinez R, Schafe G, LeDoux J, Doyére V. 2013. Detection of a temporal error triggers reconsolidation of amygdala-dependent memories. Curr Biol 23: 467-472.

Diergaarde L, Schoffelmeer A, De Vries T. 2008. Pharmacological manipulation of memory reconsolidation: Towards a novel treatment of pathogenic memories. Eur J Pharmacol 585: 453-457. 
Eisenberg M, Kobilo T, Berman D, Dudai Y. 2003. Stability of retrieved memory: Inverse correlation with trace dominance. Science 301: $1102-1104$.

Finnie P, Nader K. 2012. The role of metaplasticity mechanisms in regulating memory destabilization and reconsolidation. Neurosci Biobehav Rev 36: 1667-1707.

Flavell C, Barber D, Lee J. 2011. Behavioural memory reconsolidation of food and fear memories. Nat Commun 2: 1-9.

Gisquet-Verrier P, Riccio D. 2012. Memory reactivation effects independent of reconsolidation. Learn Mem 19: 401-409.

Golkar A, Bellander M, Olsson A, Öhman A. 2012. Are memories erasable? Reconsolidation of learned fear with fear-relevant and fear-irrelevant stimuli. Front Behav Neurosci 6: 1-9.

Hutton-Bedbrook K, McNally GP. 2013. The promises and pitfalls of retrieval-extinction procedures in preventing relapse to drug seeking. Front Psychiatry 4: 1-4.

Ishii D, Matsuzawa D, Matsuda S, Tomizawa H, Sutoh C, Shimizu E. 2012. No erasure effect of retrieval-extinction trial on fear memory in the hippocampus-independent and dependent paradigms. Neurosci Lett 523: $76-81$.

Kindt M, Soeter M. 2013. Reconsolidation in a human fear conditioning study: A test of extinction as updating mechanism. Biol Psychol 92: $43-50$.

Lee JC. 2008. Memory reconsolidation mediates the strengthening of memories by additional learning. Nat Neurosci 11: 1264-1266.

Lee J, Milton A, Everitt B. 2006. Reconsolidation and extinction of conditioned fear: Inhibition and potentiation. J Neurosci 26: 10051-10056.

Lee S, Choi J, Lee N, Lee H, Kim J, Yu N, Choi S, Lee S, Kim H, Kaang B. 2008. Synaptic protein degradation underlies destabilization of retrieved fear memory. Science 319: 1253-1256.

Lewis D. 1979. Psychobiology of active and inactive memory. Psychol Bull 86: $1054-1083$.

Ma X, Zhang J, Yu L. 2012. Post-retrieval extinction training enhances or hinders the extinction of morphine-induced conditioned place preference in rats dependent on the retrieval-extinction interval. Psychopharmacology 221: 19-26.

Mineka S, Mystkowski J, Hladek D, Rodriguez B. 1999. The effects of changing contexts on return of fear following exposure therapy for spider fear. J Consult Clin Psychol 67: 599-604.

Monfils M, Cowansage K, Klann E, LeDoux J. 2009. Extinctionreconsolidation boundaries: Key to persistent attenuation of fear memories. Science 324: 951-955.

Mystkowski J, Craske M, Echeverri A. 2002. Treatment context and return of fear in spider phobia. Behav Ther 33: 399-416.

Nader K, Hardt O. 2009. A single standard for memory: The case for reconsolidation. Nat Rev Neurosci 10: 224-234.
Oyarzún J, Lopez-Barroso D, Fuentemilla L, Cucurell D, Pedraza C, Rodriguez-Fornells A, de Diego-Balaguer R. 2012. Updating fearful memories with extinction training during reconsolidation: A human study using auditory aversive stimuli. PLoS One 7: $1-9$.

Pedreira M, Pérez-Cuesta L, Maldonado H. 2004. Mismatch between what is expected and what actually occurs triggers memory reconsolidation or extinction. Learn Mem 11: 579-585.

Pérez-Cuesta L, Maldonado H. 2009. Memory reconsolidation and extinction in the crab: Mutual exclusion or coexistence? Learn Mem 16: $714-721$.

Rao-Ruiz P, Rotaru D, van der Loo R, Mansvelder H, Stiedl O, Smit A, Spijker S. 2011. Retrieval-specific endocytosis of GluA2-AMPARs underlies adaptive reconsolidation of contextual fear. Nat Neurosci 14: 1302-1308.

Rescorla R. 1988. Behavioral studies of pavlovian conditioning. Annu Rev Neurosci 11: 329-352.

Rodríguez B, Craske M, Mineka S, Hladek D. 1999. Context-specificity of relapse: Effects of therapist and environmental context return of fear. Behav Res Ther 37: 845-862.

Schiller D, Monfils M, Raio C, Johnson D, LeDoux J, Phelps E. 2010. Preventing the return of fear in humans using reconsolidation update mechanisms. Nature 463: 49-53.

Sevenster D, Beckers T, Kindt M. 2012. Retrieval per se is not sufficient to trigger reconsolidation of human fear memory. Neurobiol Learn Mem 97: $338-345$.

Sevenster D, Beckers T, Kindt M. 2013. Prediction error governs pharmacologically induced amnesia for learned fear. Science 339: 830-833.

Soeter M, Kindt M. 2011. Disrupting reconsolidation: Pharmacological and behavioral manipulations. Learn Mem 18: 357-366.

Suzuki A, Josselyn S, Frankland P, Masushige S, Silva A, Kida S. 2004. Memory reconsolidation and extinction have distinct temporal and biochemical signatures. J Neurosci 24: 4787-4795.

Urcelay G. 2012. Exposure therapy. In Exposure therapy: Rethinking the model-refining the method (ed. Neudeck P, Wittchen H-U), pp. 35-63. Springer, New York.

Xue Y, Luo Y, Wu P, Shi H, Xue L, Chen C, Zhu W, Ding Z, Bao Y, Shi J, et al. 2012. A memory retrieval-extinction procedure to prevent drug craving and relapse. Science 336: 241-245.

Zhang J, Ma X, Yu L. 2012. Repeated paired-testing impairs extinction of morphine-induced conditioned place preference dependent on the inter-test interval in rats. Neurosci Lett 516: 72-74.

Received August 3, 2013; accepted in revised form October 6, 2013. 


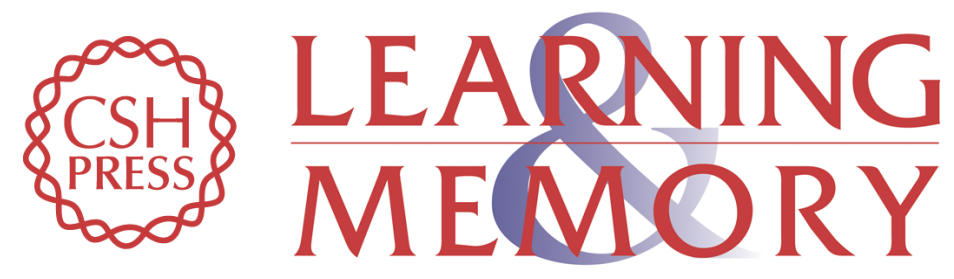

\section{Memory destabilization is critical for the success of the reactivation -extinction procedure}

Marcelo E. Piñeyro, Roque I. Ferrer Monti, Joaquín M. Alfei, et al.

Learn. Mem. 2014, 21:

Access the most recent version at doi:10.1101/Im.032714.113

\begin{aligned} & \hline References $\begin{array}{l}\text { This article cites } 46 \text { articles, } 16 \text { of which can be accessed free at: } \\ \text { http://learnmem.cshlp.org/content/21/1/46.full.html\#ref-list-1 }\end{array} \\ & \begin{array}{r}\text { Creative } \\ \text { Commons } \\ \text { License }\end{array} \begin{array}{l}\text { This article is distributed exclusively by Cold Spring Harbor Laboratory Press for the } \\ \text { first } 12 \text { months after the full-issue publication date (see } \\ \text { http://learnmem.cshlp.org/site/misc/terms.xhtml). After } 12 \text { months, it is available under } \\ \text { a Creative Commons License (Attribution-NonCommercial } 3.0 \text { Unported), as } \\ \text { described at http://creativecommons.org/licenses/by-nc/3.0/. }\end{array} \\ & \begin{array}{c}\text { Receive free email alerts when new articles cite this article - sign up in the box at the } \\ \text { top right corner of the article or click here. }\end{array} \\ & \begin{array}{l}\text { Service } \\ \text { terting }\end{array}\end{aligned}$

